\title{
A Comparative Study of Static Biofilm Formation and Antibiotic Resistant Pattern between Environmental and Clinical Isolate of Pseudomonas aeruginosa
}

\author{
Fahareen Binta Mosharraf, Sara Sadia Chowdhury, Akash Ahmed $₫$, M. Mahboob Hossain* \\ Department of Mathematics and Natural Sciences, BRAC University, Dhaka, Bangladesh \\ Email: *mmhossain@bracu.ac.bd
}

How to cite this paper: Mosharraf, F.B., Chowdhury, S.S., Ahmed, A. and Hossain, M.M. (2020) A Comparative Study of Static Biofilm Formation and Antibiotic Resistant Pattern between Environmental and Clinical Isolate of Pseudomonas aeruginosa. Advances in Microbiology, 10, 663-672.

https://doi.org/10.4236/aim.2020.1012047

Received: November 23, 2020

Accepted: December 19, 2020

Published: December 22, 2020

Copyright $\odot 2020$ by author(s) and Scientific Research Publishing Inc. This work is licensed under the Creative Commons Attribution International License (CC BY 4.0).

http://creativecommons.org/licenses/by/4.0/

\begin{abstract}
Biofilms are dense bacterial colonies, derived from microbially derived sessile community, networked within a polysaccharide matrix with a distinct architecture that has the attachment potential to both alive and abiotic surfaces. Pseudomonas aeruginosa is a model biofilm forming microorganism associated with remarkable morbidity and mortality rate due to emergence of antibiotic resistant pathogenic bacteria. Moreover, Pseudomonas aeruginosa originating from a biofilm is more resistant to a wide range of antibiotics than the planktonic bacteria. This research was planned to develop a comparative study of the biofilm production between potential, antimicrobial resistance of Pseudomonas aeruginosa isolated from mature environmental biofilm and clinical strain of the same species that did not derive from biofilm. It was observed that the Pseudomonas aeruginosa from environmental isolates were resistant to 15 prominent antibiotics, while clinical strain was comparatively resistant to only few of them. A confirmatory analysis of biofilm formation and antibiotic resistance pattern of these two groups of organisms was checked by $96-$ well microtiter plate and the disc diffusion method respectively. Finally, the results portrayed that the environmental strains with high drug resistance, potentially formed a considerable amount of biofilm in the period of a week whereas; clinical stains formed a negligible amount of biofilm within the same time frame.
\end{abstract}

\section{Keywords}

Biofilms, Clinical Isolate, Antibiotic Resistant

\section{Introduction}

Biofilm was one of the earliest discoveries during the initial phase of microbiol- 
ogy and was acknowledged as "animalculi" by the father of microbiology Antonie van Leeuwenhoek when he first observed plaque biofilm from the teeth through a microscope. It was not studied extensively until 1970s, however, biofilm aided bacteria to endure extreme heat and acidic state during the formation of earth and still continues to shelter bacteria from such severe conditions so that they can become accustomed to their extreme surroundings. Nevertheless, biofilms grasped the attention of many scientists in the recent past that made them unravel several mechanisms of these dense colonies of bacteria networked within an exopolysaccharide (EPS) matrix with a distinct architecture of bacterial communities that has the attachment potential to both living and inanimate surfaces, collectively called biofilm. Along with the EPS, there are proteins, nucleic acids, peptidoglycan, lipids, phospholipids, and other cell components present in the matrix of a biofilm community frequently known as slime [1]. Biofilms may be of a single species or of an assorted gathering of microorganisms [2]. Quorum sensing or chemical correspondence within cells of this biological system called biofilm permits the bacteria to organize their movement and form communities. Inside of a biofilm, the bacteria can share supplements and are shielded from perilous environmental factors, for example, parching, antibiotics and the immune system of the body. The nearby vicinity of microorganisms inside of a biofilm may likewise have the benefit of exchanging the hereditary data by, for instance, conjugation.

Furthermore, the EPS is engineered to provide auxiliary and defensive capacities equally. It creates channels that ease the movement of supplements, enzymes, metabolites, and transfer of wastes inside and outside the biofilm matrix [3]. Engineering is vital to multi cellular biofilms, taking into account every individual cell's prerequisites to be met to survive. The parts of the EPS and consequent layers of cells can impede the infiltration of antibiotics, encouraging the movement of supplements in and waste items out of the biofilm structure [4]. As a consequence of these attributes, the EPS lattice presents properties in biofilm related groups which are different from their planktonic partners. Biofilms are normally connected to a surface, for example, a stone in a lake, human tooth, bathroom sinks, bathtubs, medical devices, cooling towers or might be available as a flock in sewage treatment and in a filamentous streamer structure in quick flowing streams [2].

A major bacteria $P$. aeruginosa is one of the most ubiquitous biofilms forming medical device associated pathogens. In Europe, 175,000 deaths were reported due to nosocomial infections annually in 1.75 million hospitalized patients where $P$. aeruginosa is responsible for $10 \%-20 \%$ mortality cases [5]. $P$. aeruginosa is disreputably difficult to eradicate when colonizing the lungs of cystic fibrosis patients, forming thick antibiotic resistant biofilms that also guard from host immune defenses, lowering of the long-term prediction of the infected patient [6]. Other diligent diseases, for instance, unending bacterial prostatitis, endocarditis, otitis media and, periodontitis, and denture stomatitis, are brought 
about by biofilms [7]. On the other hand, biofilms also grow on sophisticated devices like urinary catheters, prosthetic heart valves, endotracheal tubes, surgical sutures, orthopedic devices, contact lenses, and dentures. This results in importunate health hazards like discomfort and inflammation.

Most research has been conducted which $P$. aeruginosa is often used as a model biofilm producing organism [8]. Biofilm formation is regulated by different genetically defined characteristics like bacterial mobility, cell membrane proteins, extracellular polysaccharides and signaling molecules and environmental factors such as presence of certain nutrients and oxygen along with level of temperature and $\mathrm{pH}$ play significant roles in biofilm formation.

Thus, it is untoward and perilous to find such an opportunistic resistant pathogen in the environment that has the potential of imposing colossal threat to the human health. To recapitulate, keeping all these in mind the present study reported the antibiotic susceptibility patterns of the environmental isolates of $P$. aeruginosa present in biofilm and also comparing them with that of the clinical strain. Reconfirming the antibiotic resistances of $P$. aeruginosa environmental isolates that were observed to form biofilm successfully and comparing them with that of the clinical strain.

\section{Materials and Methods}

\subsection{Bacterial Strain}

The research work was carried out in the Microbiology Specialized Research Laboratory of the Department of Mathematics and Natural Sciences, BRAC University, Bangladesh. In this study, a reference or clinical strain of $P$. aeruginosa was used from Dhaka Shishu Hospital, Bangladesh. For analyzing the biofilm formation capacity and drug resistance pattern a logarithmic growth phase culture of $P$. aeruginosa was preferred. An overnight bacterial culture in Luria Bertani broth (Incubation temperature: $37^{\circ} \mathrm{C}$, Incubation time: 24 hour), was harvested by centrifugation $(2500 \mathrm{rpm})$ and adjusted to the concentration required by optical density $(600 \mathrm{~nm})$. A clinical strain was identified routinely to distinguish Pseudomonas aeruginosa by streaking on nutrient agar and selective media. The cultural properties of the organism were observed and recorded. Subsequently the clinical strain was employed for biochemical confirmation tests according to the standard methods described in Microbiology Laboratory Manual (Cappuccino, J.G. and Sherman N, 1996) [9].

\subsection{Environmental Sample Collection and Processing}

Biofilm samples were collected from different sources in the month of November and December, 2015. The biofilm samples were collected from kitchen sink, bathtubs, cooling towers, rainwater pipes, machines of food industries, lake stones etc. under distinctive environmental condition (Average temperature $25.5^{\circ} \mathrm{C}$ and humidity was around $84 \%$ ). The Selected colonies (ES1 and ES2) were confirmed by following standard methods [9]. 


\subsection{Antibiotic Susceptibility Test of the Standard Clinical and Environmental Strains of Bacteria}

In antibiogram (disc diffusion) assay, the respond pattern of $P$. aeruginosa confirmed from environmental and clinical samples was investigated to record the potential antibacterial activity. In this study, the effectiveness of thirteen different antibiotics was determined. They are listed below in Table 1.

The antibiotic discs were placed on surface of MHA plates cultured with clinical and environmental isolates and incubated overnight at $37^{\circ} \mathrm{C}$ for 24 hours. Then diameter of inhibition zones was measured in $\mathrm{mm}$ (showed in Figure 1).

\subsection{Biofilm Formation}

Following the antibiotic susceptibity test, the environmental isolates and the clinical strain those showed a considerable amount of resistance against most of the antibiotics, were checked for biofilm formation. The protocol was modified and followed from Merritt, J. H. et al., (2005) [10] Selected strains of the P. aeruginosa isolated from different environmental samples and the clinical strain was grown in Luria Bertani broth to reach up to stationary phase. The raw and diluted samples $\left(1: 10,1: 100\right.$ and 1:200) were kept at $37^{\circ} \mathrm{C}$ for a week for the analysis of static biofilms by 96 -well microtiter plate biofilm assay technique. The optical density (OD) of each of these samples (the crystal violet staining of the adherent biomass) was measured at a wavelength of $620 \mathrm{~nm}$ using an ELISA machine (Finland). This experimental system is a simple high-throughput

Table 1. Provided antibiotic discs.

\begin{tabular}{cc}
\hline Ampicillin (AMP10) & Tetracycline (TE30) \\
Aztreonam (ATM30) & Amikacin (AK30) \\
Streptomycin (S10) & Ciprofloxacin (CIP5) \\
Amoxicillin (AML10) & Penicillin (P10) \\
Chloramphenicol (C30) & Erythromycin (E15) \\
Polymyxin (PB300) & Tobramycin (TOB10) \\
Gentamicin (CN10) & Kanamycin (K30) \\
Oxacillin (OX1) & \\
\hline
\end{tabular}

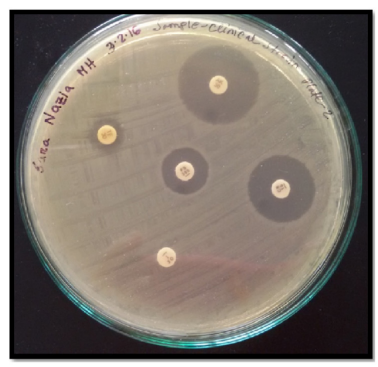

(a)

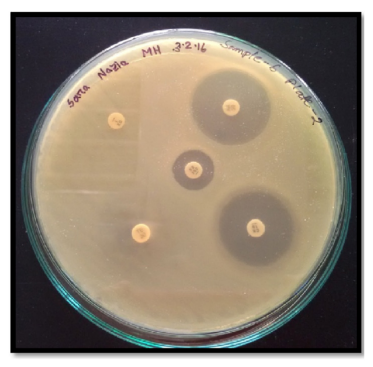

(b)

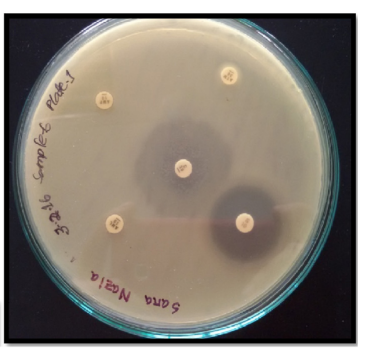

(c)

Figure 1. Zone of inhibition of the antibiotic discs on Clinical Isolate (a), Environmental Isolate 1 (b) and Environmental Isolate 2 (c). 
method used to monitor microbial attachment to an abiotic surface and forming static biofilm.

\subsection{Reconfirmation of Antibiotic Resistance by Disc Diffusion Method}

The environmental isolates of $P$. aeruginosa that could form biofilms were reconfirmed to be antibiotic resistance, by performing the antibiotic disc diffusion method. The clinical strain was also checked for antibiotic resistance alongside the environmental isolates.

Categorization of isolates based on biofilm-forming capacity.

The following criteria were used for biofilm gradation in clinical isolates as described by Hassan, A. et al., (2011) [11].

ODcut $=$ ODavg of negative control $+3 \times$ standard deviation $(\mathrm{SD})$ of ODs of negative control.

1) $\mathrm{OD} \leq$ ODcut $=$ Non-biofilm-former $(\mathrm{NBF})$

2) ODcut $\angle$ OD $\leq 2 \times$ ODcut $=$ Weak biofilm-former (WBF)

3) $2 \times$ ODcut $<$ OD $\leq 4 \times$ ODcut $=$ Moderate biofilm-former $(\mathrm{MBF})$

4) $\mathrm{OD}>4 \times$ ODcut $=$ Strong biofilm-former

\section{Result}

Identification of the bacteria was done for reconfirmation. Pseudomonas aeruginosa was reconfirmed by streaking on a selective media and by performing biochemical tests (Figure 2).

Selective antimicrobial activity test by means of Antibiogram method.

The standard disc diffusion test was done with all the provided antibiotics (Table 1) against clinical and environmental (from mature biofilm) strains of Pseudomonas aeruginosa to identify their resistance pattern (Table 2).

\subsection{The Results of Growing and Analyzing Static Biofilms by 96-Well Microtiter Plate Biofilmassay}

The static biofilms were grown with the clinical and the environmental strains. The optical density was measured using an Enzyme Linked Immunosorbent

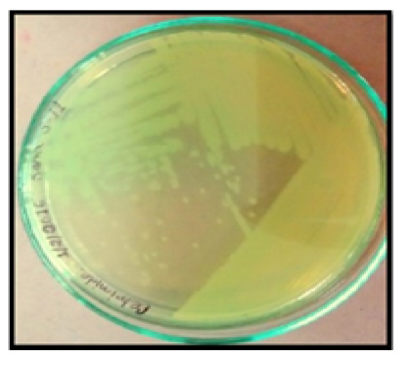

(a)

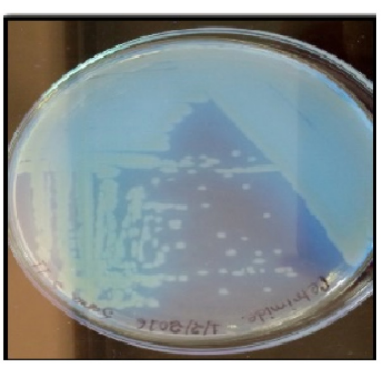

(b)

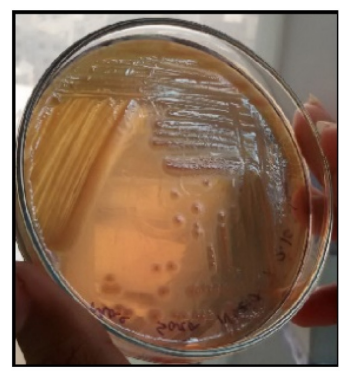

(c)

Figure 2. Cultural characteristics of clinical stain and environmental isolates on selective media. (a) P. aeruginosa in Certimide agar (b) P. aeruginosa in Cetrimide agar, viewed under UV transilluminator (c) P. aeruginosa in MacConkey agar media. 
Table 2. Selective antimicrobial activity test by disc diffusion method.

\begin{tabular}{clcl}
\hline & & P. aeruginosa & \\
\cline { 2 - 4 } Antibiotics & Clinical Sample & $\begin{array}{c}\text { Environmental } \\
\text { Isolate 1 }\end{array}$ & $\begin{array}{c}\text { Environmental } \\
\text { Isolate 2 }\end{array}$ \\
\hline Ampicillin (AMP10) & Sensitive & Resistant & Resistant \\
Aztreonam (ATM 30) & Sensitive & Resistant & Sensitive \\
Streptomycin (S10) & Sensitive & Sensitive & Sensitive \\
Amoxicillin (AML10) & Sensitive & Resistant & Resistant \\
Chloramphenicol (C30) & Sensitive & Sensitive & Resistant \\
Polymyxine (PB300) & Sensitive & Sensitive & Sensitive \\
Gentamicin (CN10) & Sensitive & Sensitive & Resistant \\
Oxacillin (OX1) & Resistant & Resistant & Resistant \\
Tetracycline (TE30) & Resistant & Resistant & Resistant \\
Amikacin (AK30) & Sensitive & Sensitive & Sensitive \\
Ciprofloxacin (CIP5) & Sensitive & Sensitive & Sensitive \\
Penicillin (P10) & Resistant & Resistant & Resistant \\
Erythromycin (E15) & Sensitive & Resistant & Resistant \\
Tobramycin (TOB 10) & Sensitive & Sensitive & Sensitive \\
Kanamycin (K30) & Sensitive & Sensitive & Resistant \\
\hline
\end{tabular}

Assay (ELISA) technique by micro ELISA autoreader. Following one week of incubation at $37^{\circ} \mathrm{C}$, the bacterial attachment was assessed by measuring the staining of the adherent biomass. The optical density of the crystal violet and acetic acid solution in each well of the optically clear flat-bottom 96-well plate was measured by spectrophotometer machine at $620 \mathrm{~nm}$. It could be worthwhile to mention that, the studies were performed in duplicates and repeated for two times for the fulfillment of the reproducibility. The negative controls wells were the uninoculated wells containing sterile MHB supplemented with $1 \%$ glucose. The negative controls wells were used as blank and the absorbance of these wells (average OD 0.0355 and standard deviation 0.000866025 ) was used to calculate $\mathrm{OD}_{\text {cut }}$ which was $0.0380 . \mathrm{OD}_{\text {cut }}$ value was used to identify whether biofilm formation of isolates exists or not. The wells showing $\mathrm{OD}$ values higher than the $\mathrm{OD}_{\text {cut }}$ considered to be biofilm producers and categorized accordingly.

The result revealed that drug resistant $P$. aeruginosa selected from mature environmental raw sample formed static biofilm in microtiter plate (average OD for ES1 2.817 and ES2 2.518 and their standard deviation was 0.009 and 0.0495 respectively). The gradual decrease of both environmental samples (ES1 and ES2) in average OD for dilution 1:10 $(0.85,0.45), 1: 100(0.27,0.19) \& 1: 200$ $(0.11,0.082)$ was significantly correlated to the dilution of that sample. In an attempt to determine the most efficacious biofilm forming strain, the clinical strain of antibiotic resistant $P$. aeruginosa was kept for the same time frame and showed least potential capacity of biofilm formation reported by OD measurement. The average OD for raw clinical sample was 0.0395 which showed insigni- 
ficant variation in diluted samples and coincides with the negative control group (Figure 3). The experiment was not prolonged after a week to avoid the possibility of contamination with other prevailing microorganisms which would have interfered with the actual picture.

\subsection{Categorization of Isolates Based on Biofilm-Forming Capacity}

The study results finally revealed that the clinical isolate of $P$. aeruginosa was not a potential biofilm former in raw or any other dilution whereas both of the environmental samples of $P$. aeruginosa (ES1 and ES2) were strong biofilm former up to 1:100 dilution. The dilution 1:200 of the both ES1 and ES2 were found to be moderate biofilm former.

\section{Discussion}

Biofilm-related infections signify one of the imperative concerns of contemporary medicine due to their abilities to withstand antibiotics, host defense systems and other external stresses; therefore, responsible for developing persistent chronic infections. To control biofilm formation or eliminate mature biofilm from natural environment several control strategies are applied. The present report will focus on the comparative analysis of antibiotic resistance pattern of one of the landmark biofilms forming organism Pseudomonas aeruginosa isolated from environmental mature biofilm and clinical samples.

To begin with, the biofilm sources were chosen to be kitchen sink, bathtubs, cooling towers, rainwater pipes, machines of food industries, lake stones, etc. A clinical strain of $P$. aeruginosa was also obtained from Dhaka Shishu hospital,

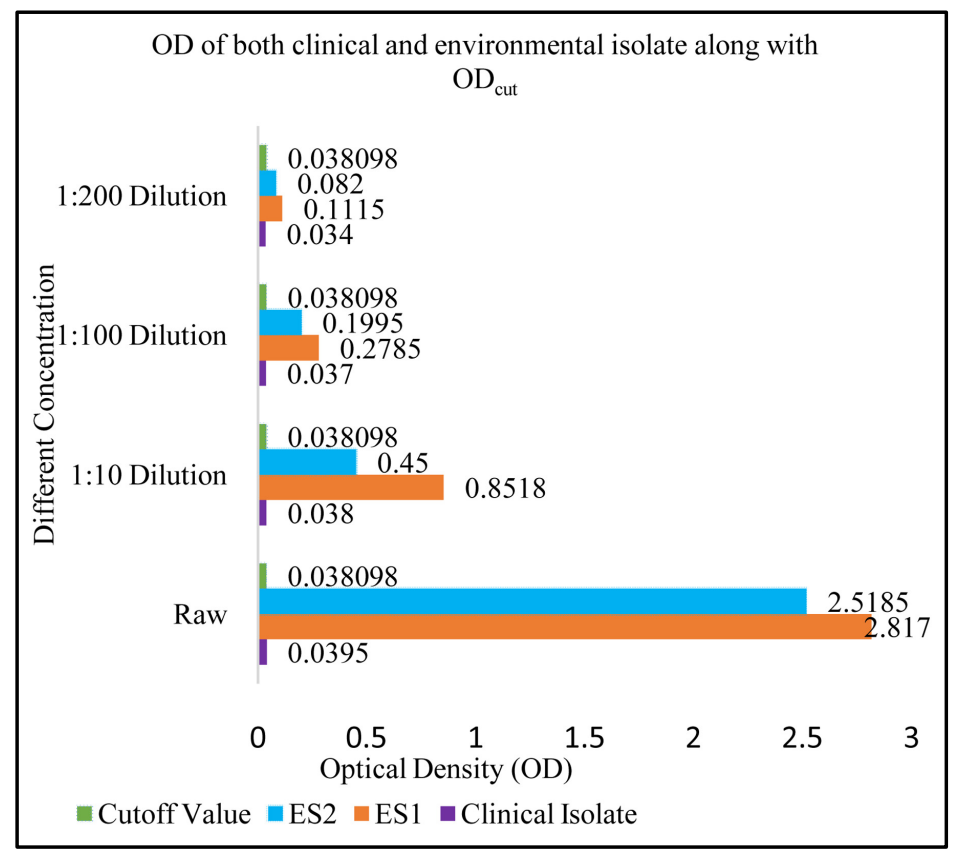

Figure 3. The graphical presentation of OD of clinical and environmental samples of $P$. aeruginosa compared to $\mathrm{OD}_{\text {cut }}$. 
Bangladesh, which worked as a reference strain. Specific $P$. aeruginosa strains from mature biofilm and clinical samples were isolated and identified by streaking on nutrient agar and selective media, and the cultural properties of the organism was observed and recorded (Figure 2). For further confirmation of the strains to be $P$. aeruginosa, various physical and biochemical tests were performed with the environmental isolates and clinical strain and it was assured that all the tests gave the exact results that are standard for $P$. aeruginosa [12].

The antibiotic susceptibility test was done with the prominent antibiotic discs (Table 1) and diameter of zone of inhibition was observed and measured (Figure 1) and compared to the chart provided by National Committee for Clinical Laboratory Standards (NCCLS). According to the results, the environmental biofilm isolates of $P$. aeruginosa showed more prompt results as compared to the clinical strain. Out of the 15 antibiotics the clinical strains were resistant against only 3 antibiotics, Oxacillin, Tetracycline and Penicillin. Whereas, the environmental isolates were found to be resistant against up to 7 to 10 antibiotics namely, Ampicillin, Aztreonam, Amoxicillin and Chloramphenicol, Gentamicin, Oxacillin, Tetracycline, Penicillin, Erythromycin and Kanamycin. This correlates with point which was mentioned earlier that $P$. aeruginosa originating from biofilm can gain resistances to antibiotics due to a lot of factors, just as, counting alteration of the anti-infection target site, efflux pumps, the option of utilizing a wide variety of metabolic pathways to keep away from antibiotic target, and secretion of enzymes that wreck the antibiotics, etc. To emphasize, the chromosomal Amp C Cephalosporinase, the outer membrane porin Opr D, and the multitude of efflux pumps, and the complex mechanisms and pathways by which $P$. aeruginosa regulates and correlates their expression, are the key reasons of its antibiotic resistance [13].

To reconfirm the potential biofilm forming $P$. aeruginosa, 96-well microtiter plate biofilm assay technique was performed. This method is usually implemented to study bacterial biofilm as it is a vital means of examining the initial phases of biofilm formation. Nevertheless, the assay has been successful at identifying many aspects needed in initiating biofilm formation and as well as genes concerned with extracellularpolysaccharide production. Certainly, biofilms grown in microtiter dishes successfully carry the properties of a mature biofilm, such as antibiotic tolerance and resistance to immune system effectors etc. [14]. The clinical and environmental isolates of $P$. aeruginosa those showed resistance to the highest number of antibiotics were selected to prove their potential to establish biofilms within a period of one week. The biofilm formed within this period were stained with crystal violet and the optical density was measured using an ELISA machine at $620 \mathrm{~nm}$ for the quantification of biofilm formation which is a logarithmic function and increasing the number of light absorption units i.e. the optical density is directly proportional to the biofilm formed.

By comparing these outcomes, the ELISA results revealed the fact that (Figure 3), $P$. aeruginosa strains isolated from the environmental sample had the capability of forming biofilm and the amount of biofilm formed is observed to de- 
crease with the decreasing bacterial concentration. In contrast, the optical density values of $P$. aeruginosa clinical strain were negligible as they were close to the optical density values of the negative control. However, the trend of decreased biofilm formation with decreasing bacterial concentration was observed to be the similar in both cases.

Hence, to ensure reproducibility the antibiotic resistant environmental isolates of $P$. aeruginosa those successfully formed biofilm were subjected to antibiotic susceptibility test against the same batch of antibiotics for confirming their resistances. Eventually identical outcome ensured the biofilm mediated P. aeruginosa is more resistant than the one that does not originate from biofilm. According to the National Institutes of Health, $80 \%$ of all infections in humans are related to biofilms [15] and at present, biofilms are so widespread in the environment that it is existent starting from industrial settings like sewage treatment plants and food industries to delicate medical devices. To worsen the situation, biofilm forming opportunistic human pathogens such as $P$. aeruginosa is increasingly exhibiting resistance to multiple antibiotics verified in this present study. Continued increases in immunosuppressed patient group and the evolutionary benefits of bacteria to hastily mutate and adapt to antibacterial threats in their environment make the treatment of infectious diseases a deliberate challenge. Moreover, the irresponsible and improper utilization of antimicrobials also elevated the resistant strains in place of the sensitive ones [16], and it is legitimate for the opportunistic pathogen $P$. aeruginosa since, it holds the capacity to be multidrug-resistant.

\section{Conclusion}

The biofilm inhabitation of these antibiotic resistant $P$. aeruginosa is proving to be a menace to the society. To conclude, from the findings in this study, the $P$. aeruginosa environmental isolates formed biofilms and were observed to be resistant to most of the provided antibiotics compared to the clinical strain that can hardly form biofilm and was comparatively susceptible to more antibiotics. Thus, these biofilms forming antibiotic resistant strains of $P$. aeruginosa are proved to be a threat to the human community. Yet, sheer implementation of careful and adequate surveillance strategies by humans can eradicate the incidence of towering mortality and morbidity related to infections of $P$. aeruginosa associated with biofilms.

\section{Acknowledgements}

Prof. Mahbubul Alam Majumdar, Interim Dean, School of Sciences, BRAC University. Prof. A.F.M. Yusuf Haider, Chairperson, Department of Mathematics \& Natural Sciences, Brac University, Dhaka, Bangladesh. Dr. Mahbubul Hasan Siddiquee, Coordinator, Microbiology Program, BRAC University.

\section{Conflicts of Interest}

The authors declare no conflicts of interest regarding the publication of this paper. 


\section{References}

[1] Sutherland, I.W. (2001) The Biofilm Matrix-An Immobilized but Dynamic Microbial Environment. Trends in Microbiology, 9, 222-227. https://doi.org/10.1016/s0966-842x(01)02012-1

[2] Tortora, G.J., Funke, B.R. and Case, C.L. (2012) Microbiology: An Introduction. Pearson, London.

[3] Stewart, P.S. and Costerton, J.W. (2001) Antibiotic Resistance of Bacteria in Biofilms. Lancet, 358, 135-138. https://doi.org/10.1016/S0140-6736(01)05321-1

[4] Simões, M., Bennett, R.N. and Rosa, E.A. (2009) Understanding Antimicrobial Activities of Phytochemicals against Multidrug Resistant Bacteria and Biofilms. Natural Product Reports, 26, 746-757. https://doi.org/10.1039/B821648G

[5] Laverty, G., Gorman, S.P. and Gilmore, B.F. (2014) Biomolecular Mechanisms of Pseudomonas aeruginosa and Escherichia coli Biofilm Formation. Pathogens, 3, 596-632. https://doi.org/10.3390/pathogens3030596

[6] Smith, K. and Hunter, I.S. (2008) Efficacy of Common Hospital Biocides with Biofilms of Multi-Drug Resistant Clinical Isolates. Journal of Medical Microbiology, 57, 966-973. https://doi.org/10.1099/jmm.0.47668-0

[7] De Carvalho, C.C. (2007) Biofilms: Recent Developments on an Old Battle. Recent Patents on Biotechnology, 1, 49-57. https://doi.org/10.2174/187220807779813965

[8] McDougald, D., Klebensberger, J., Tolker-Nielsen, T., Webb, J.S., Conibear, T., Rice, S.A., Kirov, S.M., Matz, C. and Kjelleberg, S. (2008) Pseudomonas aeruginosa: A Model for Biofilm Formation. In: Rehm, B.H.A., Ed., Cell Factory, Wiley-VCH Verlag GmbH \& Co. KGaA, Weinheim, 215.

[9] Cappuccino, J.G. and Sherman, N. (2013) Microbiology: A Laboratory Manual. 10th Edition, Pearson Education Limited, London.

[10] Merritt, J.H., Kadouri, D.E. and O’Toole, G.A. (2005) Growing and Analyzing Static Biofilms. Current Protocols in Microbiology, 00, 1B.1.1-1B.1.17. https://doi.org/10.1002/9780471729259.mc01b01s00

[11] Afreenish, H., Javaid, U., Fatima, K., Maria, O., Ali, K. and Muhammad, I. (2011) Evaluation of Different Detection Methods of Biofilm Formation in the Clinical Isolates. Brazilian Journal of Infectious Diseases, 15, 305-311. https://doi.org/10.1016/S1413-8670(11)70197-0

[12] Arai, T., Otake, M., Enomoto, S., Goto, S. and Kuwahara, S. (1970) Determination of Pseudomonas aeruginosa by Biochemical Test Methods. Japanese Journal of Microbiology, 14, 279-284. https://doi.org/10.1111/j.1348-0421.1970.tb00525.x

[13] Lister, P.D., Wolter, D.J. and Hanson, N.D. (2009) Antibacterial-Resistant Pseudomonas aeruginosa: Clinical Impact and Complex Regulation of Chromosomally Encoded Resistance Mechanisms. Clinical Microbiology Reviews, 22, 582-610. https://doi.org/10.1128/CMR.00040-09

[14] O’Toole, G.A. (2011) Microtiter Dish Biofilm Formation Assay. Journal of Visualized Experiments, No.47, e2437. https://dx.doi.org/10.3791/2437

[15] Miquel, S., Lagrafeuille, R., Souweine, B. and Forestier, C. (2016) Anti-Biofilm Activity as a Health Issue. Frontiers in Microbiology, 7, 592. https://doi.org/10.3389/fmicb.2016.00592

[16] Hellinger, W.C. (2000) Confronting the Problem of Increasing Antibiotic Resistance. Southern Medical Journal, 93, 842-848. https://doi.org/10.1097/00007611-200009000-00001 